\section{Impact of direct electronic optometric referral with ocular imaging to a hospital eye service}

JR Cameron, S Ahmed, P Curry, G Forrest and $R$ Sanders
Department of

Ophthalmology, Queen Margaret Hospital, Dunfermline, Fife, UK

Correspondence: JR Cameron, Department of Ophthalmology, Queen Margaret Hospital, Whitefield Road, Dunfermline, Fife, KY12 OSU, UK

Tel: + 441383623 623; Fax: + 448701369737.

E-mail: j.cameron@ nhs.net

Received: 30 August 2007 Accepted in revised form: 31 May 2008 Published online: 4 July 2008

Competing interests: None

The study was funded by the Electronic Clinical and Communications Implementation project (Scottish government)

\begin{abstract}
Aims A study to assess the feasibility, safety, and clinical effectiveness of electronic referral — with and without images — of patients directly from optometrists in primary care to the hospital eye service (HES) in contrast to the traditional paper-based referral, through the general practitioner (GP).

Methods Three optometry practices sent consecutive referrals with images through the NHS Net to the HES. The standard General Ophthalmic Service form was electronically redesigned with additional information on patient choice for advice, appointment, or surgery. All paper referrals to the HES from the same three optometry practices before the study period were analysed (control group A) as were all paper referrals from the remaining optometrists in Fife (control group B).

Results A total of 346 electronic referrals were received over 18 months. 218 (63\%) were classified as requiring and $128(37 \%)$ as not requiring a HES appointment. The latter were subsequently examined with unexpected pathology found in three cases (glaucoma, macular pigment epithelial detachment, and possible peripheral retinal tear). In both groups, the major pathologies reported were macular degeneration, cataract, glaucoma, diabetic retinopathy, and abnormal retinal appearances. A total of $17(\mathbf{1 5 \%})$ patients in group $A$ and $26(8.4 \%)$ patients in group $B$ were classified as not requiring HES appointment. These control groups indicate that approximately $10-15 \%$ of paper referrals are not seen in the HES. To summarise, therefore, $63 \%$ of people referred by the optometrist directly using electronic referral (with or without images) were given a HES appointment compared to $85 \%$ of people
\end{abstract}

referred through the traditional paper method (without images) through their GP.

Conclusion Electronic referral with images to the HES is safe, speedy, efficient, and clinically accurate given some limitations and avoids unnecessary consultation in $37 \%$ of referrals. Eye (2009) 23, 1134-1140; doi:10.1038/eye.2008.196; published online 4 July 2008

Keywords: eHealth; electronic referral; ocular imaging

\section{Introduction}

The Scottish government is committed to the ongoing modernisation of the NHS services with the support of innovative information technology (IT) projects. ${ }^{1}$ The Electronic Clinical Communications Implementation (ECCI) is an established programme of IT development within Scotland, with an objective to facilitate electronic communication between primary and secondary care. Their remit includes referrals, appointment booking, obtaining test results, and transmitting discharge letters. ${ }^{2}$

The Queen Margaret is a 949-bedded new hospital built to serve a population of 400000 . At inception, a uniform IT system was installed throughout the hospital to support both administrative and clinical needs. Every clinical space in the hospital is computerised with an on call dedicated IT support team. Using the hospital IT system, ophthalmology has benefited from a uniform patient administration system, glaucoma, ${ }^{3}$ uveitis and macular patient electronic records, and cataract, paediatric, and diabetic audit collection.

In 2005, with the background knowledge of IT success and innovation within the hospital, the 
Scottish government, through the ECCI, funded a pilot project to evaluate the feasibility, safety, clinical effectiveness, and cost of electronic referral with images of patients directly from the optometrist to the Hospital Eye Service (HES) with concomitant information of referral to the general practitioner (GP). This paper reports our experience to date. The project was discussed and agreed with the Fife, Forth Valley and Tayside Research Ethics Service.

\section{Materials and methods}

Three optometry practices already equipped with fundus imaging cameras, agreed to participate in this prospective controlled study. All had Topcon NW6 non-mydriatic cameras that capture 45 degrees of fundus with a possible further 45 degrees by altering to peripheral fixation. The images were then formatted as either JPG ${ }^{\circledR}$ or DICOM ${ }^{\circledR}$ images and sent as email attachments using Microsoft Outlook ${ }^{\mathbb{R}}$ through the NHS Net. The NHS Net was utilised as this already has BMA approval for transmitting confidential clinical communication. ${ }^{4}$ The ECCI financed the broadband connection for the three practices at a cost of $£ 240$ per annum. The optometrists provided a patient information leaflet and consented patients into the study, with a copy sent to the patient's GP. The referral was accompanied by an image, if appropriate. The patient was charged $£ 15$ for photography by the optometrist. The information letter clearly stated that the new electronic system of referral was a pilot study, and although it may not directly benefit the current consultation, it had the potential to benefit other patients and future consultations. It also informed the patient that they would have a letter from the hospital within 6 weeks with information on the outcome of the referral. There was also explanation that for those deemed not needing to be seen, an appointment would still be sent to verify the clinical findings. On GP receipt of the optometry referral, a medical and drug history for the patient was sent through the Scottish clinical information (SCI) gateway to the HES.

A new electronic referral form was designed in collaboration with the optometrists and consultant ophthalmologists within the department. The new form contained sections for all the information previously collected in the general ophthalmic services form (GOS 18) but also required the optometrist to specify more accurately the main patient complaint and clinical diagnosis. In addition, details on the patient's last HES appointment as well as the patient's preference for surgery, hospital appointment, or advice were also incorporated. This was felt important by all consultants, as it informed the ophthalmologist of patient expectation. Patient preference was not acted upon by the optometrist but rather the clinical indication for the HES referral as before. There was also strict agreement with the optometrists that the facility to electronically refer would only be used for consecutive referrals as previously with paper referrals, and should not be abused for unnecessary

communication.

Each referral was analysed by a consultant ophthalmologist (RS) whose assessment was based solely on the information provided within the referral itself. Following assessment, they were each classified as either requiring or not requiring a HES appointment. The decision, along with supporting reasons, was communicated back to the referring optometrist by email, and by letter to the patient and GP.

Flowcharts of old and new referral pathways

\begin{tabular}{cc}
\hline Old (2-32 weeks) & New (1-6 weeks) \\
$\begin{array}{cc}\downarrow \\
\text { Optometrist appointment }\end{array}$ & $\begin{array}{c}\text { Optometrist appointment } \\
\downarrow\end{array}$ \\
GP's letter to hospital & $\begin{array}{c}\text { Electronic referral to HES with } \\
\text { images and concomitant } \\
\text { information to GP }\end{array}$ \\
$\downarrow$ & $\begin{array}{c}\downarrow \\
\text { General hospital medical }\end{array}$ \\
records & Consultant review of referral \\
$\downarrow$ & and images \\
$\downarrow$ & \\
Consultant referral file & HES appointment or discharge \\
$\downarrow$ & \\
Awaiting further information/ & \\
case notes / transfer to another & \\
consultant & \\
$\downarrow$ & \\
HES appointment
\end{tabular}

To validate this method of screening referrals and to ensure safety, all patients including those deemed not requiring a HES appointment were subsequently invited to attend the HES within 2 months of original referral, where they were seen by one of the two ophthalmologists (JC and SA). Here, they were treated as new referrals and the examiners blinded to the original assessment of the referral by RS. These two ophthalmologists had no access to patient images and had to come to a clinical decision based on a full ophthalmological examination.

The patients deemed not requiring a HES appointment also underwent a telephone interview in May 2007 regarding the service they had received. The patients were also analysed in the hospital electronic patient administration system to detect whether they had subsequently been seen in the HES after their electronic referral. 


\section{Comparison and control groups}

\section{Group A}

To examine how direct electronic optometric referral varied from current practice, it was important to assess the current 'gate-keeping' role of the GP. The traditional practice in Fife is that the optometrists will send a copy of the GOS 18 form to the GP who then places the optometrist's findings in the context of the patient's medical history and forwards the whole referral to the HES. Occasionally, the GP will choose to see the patient themselves and manage the ophthalmic problem in practice.

We, therefore, retrospectively examined all paper referrals from the same three optometry practices, in the 6 months before the commencement of our study (January-June 2005), which were passed to the GP. We investigated them to assess what proportion was referred on or not to the HES. This formed our control group.

\section{Group B}

A second comparison group that we examined was all paper referrals from all optometrists in Fife to the participating consultant only, over a 6-month period (July-December 2006). This enabled us to calculate the rate of paper-based referrals the consultant was presently marking as 'not to be seen' to establish comparison of the effect of referring electronically with images. This also allowed us comparison of the three optometry practices in the study with all optometry practices in Fife, with regards to the outcome of optometry paper referral to the HES.

\section{Results}

During the study period of 18 months from July 2005 to January 2007, 346 consecutive electronic referrals were received. No patient was omitted due to poor quality imaging and all patients in whom imaging was deemed necessary gave consent and accepted the charge of $£ 15.00$. One patient could not be imaged due to kyphoscoliosis. The age range was from 5 to 93 years old, with a mean age of 69 and a male/female ratio of 5:8. Of these 346 referrals, 218 (63\%) were classified as requiring and $128(37 \%)$ as not requiring a HES appointment. There was a very small increase in the number of referrals by the electronic system-346 electronic referrals over 18 months compared to 112 paper referrals over 6 months. This is equivalent to an increase of 10 referrals in 18 months $(2.9 \%)$

Table 1 outlines the diagnosis categories in the 218 patients that were seen in the HES. In this group, 160 $(73 \%)$ had attached electronic images.
Table 2 outlines the diagnosis categories in the 128 patients that were assessed to not require a HES appointment. In this group, 119 (93\%) had attached electronic images.

These 128 patients were subsequently invited to attend a HES appointment where they had a full ophthalmic examination. There was full agreement between electronic and clinic assessment apart from the following four cases and details of those cases are outlined below.

1. A patient with enlarged optic disc cups actually appeared more suspicious in clinic, though intraocular pressures and subsequent computerised fields were normal and the patient was discharged.

Table 1 Primary diagnoses in the 'seen' group of patients

\begin{tabular}{lc}
\hline Diagnosis & Number \\
\hline Cataract & 86 \\
Glaucoma & 26 \\
Age-related macular degeneration (ARMD) & 25 \\
Retinal artery/vein occlusion & 11 \\
Conjunctival/corneal abnormalities & 11 \\
Neuro/motility disorder & 11 \\
PVD/retinoschisis/macular hole & 10 \\
Diabetic retinopathy & 8 \\
Lid cysts/malpositions & 6 \\
Naevus & 5 \\
Postoperative problems & 4 \\
Ptosis & 3 \\
Retinal dystrophy & 2 \\
Uveitis & 2 \\
Central serous retinopathy/macular oedema & 2 \\
Blepharospasm & 2 \\
Benign floaters & 2 \\
Unknown or nil & 2 \\
\hline
\end{tabular}

Table 2 Primary diagnoses in the 'not seen' group of patients

Diagnosis Number

Dry ARMD with vision $<6 / 60$

Diabetic retinopathy (non-proliferative) 15

Corneal/conjunctival abnormalities 14

Cupped discs/glaucoma suspect 12

Isolated retinal haemorrhage 10

Cataract with good vision $\quad 9$

$\begin{array}{ll}\text { Normal appearances } & 7\end{array}$

Retinoschisis/epiretinal membrane $\quad 5$

Choroidal naevus 4

Lid cyst 4

Diplopia 3

Posterior vitreous detachment 3

Old retinal scar 3

Normal postoperative appearances 2

Small branch vein occlusion 1

Amblyopia 
2. A patient classified as dry age-related macular degeneration (ARMD) only was also found to have cupped discs with visual field defects.

3. A case deemed as dry ARMD had a small pigment epithelial detachment within the dry ARMD. This settled with observation and required no further treatment.

4. A patient with a history of bilateral cataract surgery and capsulotomies had vague symptoms of visual disturbance with no deterioration in visual acuity. Images of the anterior segment and posterior pole showed no abnormality. The extreme retinal periphery was not visualised on the digital image. Six weeks following electronic referral (and before the patient could be seen for verification of any treatable disease) the patient re-presented to the same optometrist with specific symptoms of flashes and floaters. Examination revealed a peripheral retinal detachment. The patient was referred to a vitreoretinal surgeon and treated successfully.

\section{Comparison group $A$}

Within 6 months before the commencement of our study, 112 referrals were sent from the same three optometry practices through the GP. Of these 112, six (5\%) were not referred on to the HES by the GP.

Of the 106 referred on, these were vetted by all six consultants within the ophthalmology department, who classified $95(90 \%)$ referrals as requiring a HES appointment. Thus, of the total original 112 referrals from the three optometrists to GP, 95 (85\%) were classified as requiring a HES appointment.

\section{Comparison group $B$}

This was a retrospective analysis of all paper referrals vetted by RS personally, from any optometrist in Fife, over a 6-month period between July and December 2006. A total of 309 referrals were sent to RS, of which 283 (92\%) were classified as requiring a HES appointment.

Of the 128 patients in our study classified as not requiring a HES appointment, 114 were contacted by telephone in May 2007 (eight patients had died and six patients were not contactable). Three patients indicated that they preferred a hospital consultation for greater reassurance. The rest were extremely positive about the new referral pathway. Of these 128 patients, 10 (7.8\%) were seen again in the HES between July 2005 and January 2007. Four patients were seen for pathology detected during the study, as described previously, three for unrelated disease (corneal abrasion, marginal keratitis and uveitis), and three for progression of disease (cataract and pterygium).

\section{Discussion}

We are encouraged by the results of our direct electronic referral system with ocular images. The obvious benefits are speedier clinical decision making for patients with avoidance of unnecessary hospital appointments and a higher level of interprofessional communication and feedback with optometrists than we have had hitherto.

\section{Cooperation with GPs and optometrists}

Good cooperation and communication with our GPs and optometrists and redesign of our current referral form were fundamental to the success of the new scheme. Like others, we were dissatisfied with the current GOS 18 referral form, which has been shown to suffer from incompleteness and unclear indication for referral. ${ }^{5} \mathrm{It}$ may be that the redesigning of the referral form contributed to reduce unnecessary HES appointments, along with our principal intervention of direct optometric electronic referral. The concept of direct optometric referral with concomitant information to the GP was discussed and passed through the GP local negotiating committee.

It would have been more ideal had we been able to randomly allocate optometric practices to the study, but this was not possible at the outset of the study.

\section{Limitations of the system}

The limitations of the system are well demonstrated by the four cases that were deemed not requiring a HES appointment and subsequently found to have pathology. The optometrist camera systems did not image the extreme retinal periphery and, therefore, we cannot be certain that in case four, a peripheral retinal tear was not missed at the point of electronic referral. In case one, the referral pointed to macular disease, but in fact at clinic, the patient was noted to have cupped discs and visual field defects. This is perhaps the risk associated with, in essence, conducting a 'virtual clinic' without the benefit of patient contact and therefore making a significant clinical oversight when dealing with purely electronic images and information. Case two, where there was a difference in opinion of the appearance of a set of optic discs, is not an unusual scenario and, strictly speaking, should not be a part of this group.

\section{Macular disease}

This group totalled 60 (20\%) of the 346 electronic referrals, therefore, represents a substantial proportion. This contrasts with an analysis of new patients conducted at Moorfields hospital in 1997 when macular 
diseases was responsible for $6 \%$ of all referrals. ${ }^{6}$ The change is most likely to be linked to the new treatment options available.

Of these 60 cases, $25(42 \%)$ were allocated a HES appointment and $35(58 \%)$ were not. In the latter group, one patient (case three) had a small pigment epithelial detachment within an area of dry ARMD. We feel that when diagnosis is crucially dependent on fine threedimensional visualisation, maximum caution is required in clinical decision making, taking into consideration patient symptoms and subtle changes in vision.

With the advent of new macular treatments that are time sensitive, macular disease overtly lends itself to speedy referral schemes with imaging. A recent study suggests that non-stereo digital fundus images are safe and valid for screening for ARMD. ${ }^{7}$ A further report demonstrated that electronic referral of patients with ARMD accompanied by digital images significantly reduced referral delays, speeding up access to photodynamic therapy treatment, and resulting in improved visual outcome. ${ }^{8}$

\section{Patient satisfaction}

The positive feedback and comments from the telephone interview assessment of our patients, from those deemed not requiring a HES appointment, gives further validity to our new scheme. The rate of patients failing to attend a new hospital appointment in an ophthalmic department is known to be approximately $30 \%^{9}$ and contributes to unnecessary wastage of limited NHS resource. Elderly patients have difficulty with transport, appointment instructions and hold differing perceptions of need for HES appointments. The latter is even more relevant when the optometrist is under obligation to send a referral when an abnormality is noted but the patient is asymptomatic. It is also known that $50 \%$ of new ophthalmic outpatients are discharged at their first appointment. ${ }^{6}$ Given this existing situation, electronic referral with images has obvious benefits for the patients and reassures the optometrist that they may continue to monitor the patients in the community.

\section{Financial saving}

A highly significant outcome was that 128 (37\%) patients were assessed as not requiring a HES appointment. Our comparison control groups showed that GPs manage approximately $5 \%$ of optometry referrals themselves and that consultants do not allocate HES appointments for approximately $8-10 \%$ of paper referrals. Over and above

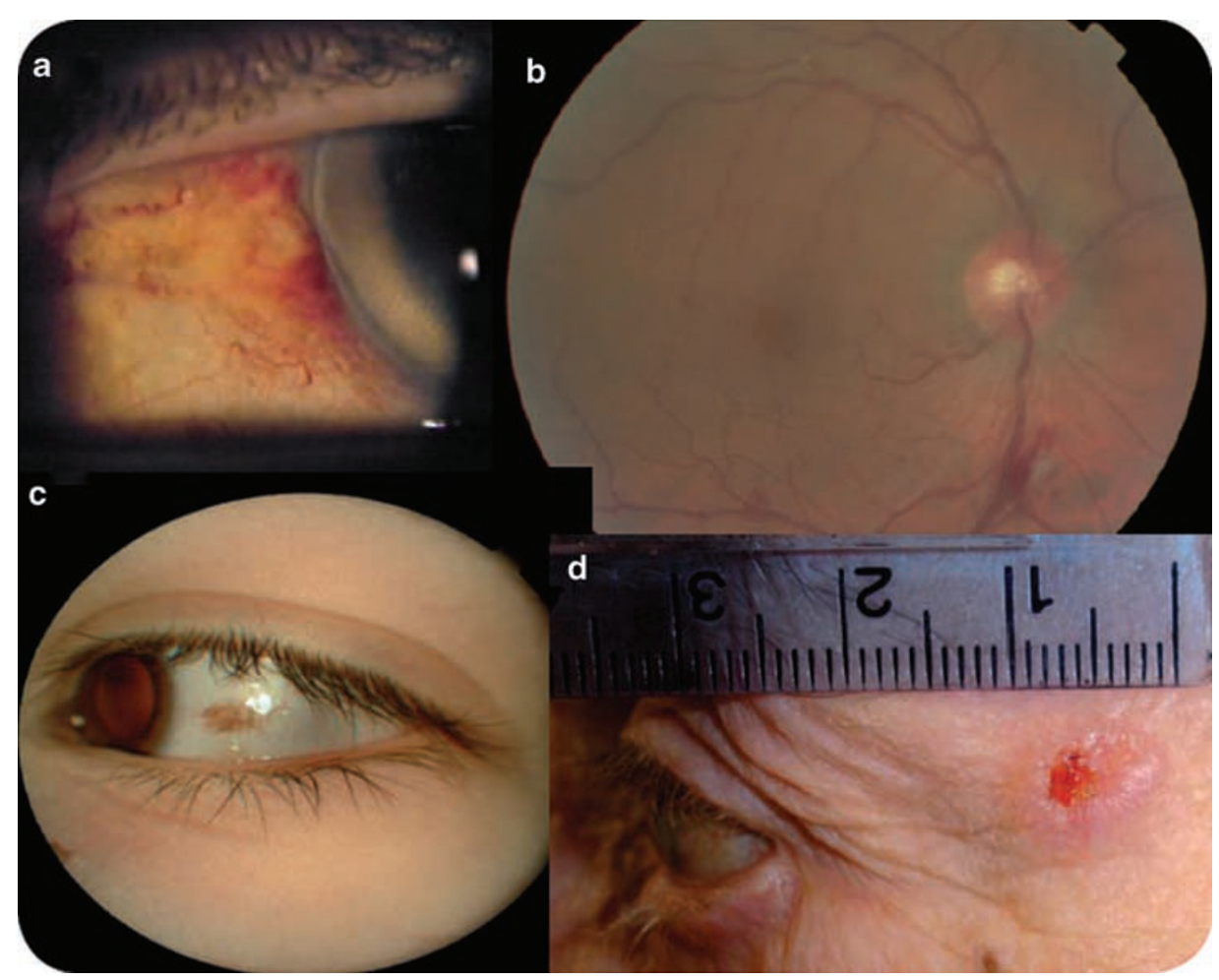

Figure 1 Examples of images sent with referrals to assist in patient assessment/diagnosis. (a) Shows a small area of inflammation around the paracentesis site, following cataract surgery - not seen. (b) Shows a branch retinal vein occlusion-seen urgently. (c) Shows a conjunctival naevus - not seen. (d) Shows a skin basal cell carcinoma - seen urgently. 
this, electronic referrals with images would appear to save a further $22 \%$ of new HES appointments.

With the published cost of outpatient appointments in Scotland ranging from $£ 108-£ 307$ per appointment, ${ }^{10}$ the extrapolation for potential savings runs into several hundred thousands of pounds per annum for individual hospital trusts.

The counter balance to this financial saving is that consultant assessment of the referrals and images is in fact a 'virtual clinic', with an assessment being made of the patient, without the patient's physical presence. Such assessments do require consultant time and secretarial support in the same way as outpatient clinics do. Much has been written about the cost effectiveness of electronic referral systems, such as a national study in Denmark ${ }^{11}$ and teledermatology services in Norway ${ }^{12}$ and the United Kingdom. ${ }^{13}$ Our study was not designed to assess costeffectiveness; however, there are savings clearly to be made with the utilisation of electronic systems such as this.

\section{Other ophthalmology/medical systems}

A similar referral scheme in dermatology demonstrated that $25 \%$ of patients referred did not need a hospital appointment, on the basis of the referral with imaging. ${ }^{14}$

One region has described its successful experience with integrating electronic referrals with their appointment booking system leading to reduced hospital appointments. ${ }^{15}$

Teleophthalmology has been successfully used to triage emergency eye conditions in rural Australia ${ }^{16}$ reducing the number of hospital referrals and preserving patient satisfaction. ${ }^{17}$ It has also been shown that remote interpretation of images to diagnose retinopathy of prematurity (ROP) is highly accurate among multiple readers for the detection of ROP requiring treatment. ${ }^{18}$ And of course, diabetic retinopathy photographic screening is an established and validated programme of disease diagnosis and management based on community-acquired photography (Figure 1).

\section{Conclusion}

In summary, this is a study showing that electronic optometric referral with images to a HES is safe, speedy, efficient, and clinically accurate given some limitations and avoids unnecessary HES consultations. Patients are satisfied with community care and the scheme also embraces the new ways of working in close collaboration with optometry colleagues. ${ }^{19}$ The Scottish government is in the process of placing all optometrists on the NHS Net and issuing grants for imaging equipment.

If this scheme is extended to all optometrists, it has the potential for considerable financial saving within an NHS ophthalmic department and shortens waiting times for new patients that specifically require hospital expertise and appointments.

Locally, we have developed a Central Ophthalmic Electronic Referral Unit (COERU) with $80 \%$ of our local GPs and optometrists computerised, and all in favour of the new scheme.

We believe our experience serves as an example of the benefits of electronic referral with imaging to an ophthalmic department.

\section{Acknowledgements}

We are grateful to Colin Ferrier, Kirit Rach and Charles MacKinnon whose optometry practices took part in the study.

\section{References}

1 Building A Health Service Fit For The Future - Volume 2: A guide for the NHS; . Online at http:// www.scotland.gov.uk/Publications/2005/05/23141500/ 15035. publication date 25 May 2005. ISBN 075594545 X.

2 ECCI national website -. http://www.nhsfife.scot.nhs.uk/ ecci/optometry.

3 Imrie F, Blaikie A, Cobb C, Sinclair A, Wilson D, Dobson S et al Glaucoma electronic patient record-design, experience and study of high-risk patients. Eye 2005; 19: 956-962.

4 Using NHS mail for Clinical Communications. BMA and NHSIA 2005.

5 Lash SC. Assessment of information included on the GOS 18 referral form used by optometrists. Ophthal Physiol Opt 2003; 23: 21-23.

6 Claoue C, Foss A, Daniel R, Cololing B. Why are new patients coming to the eye clinic? An analysis of the relative frequencies of ophthalmic disease amongst new patients attending hospital eye clinics in two separate locations. Eye 1997; 11: 865-868.

7 Jain S, Hamada S, Membrey WL, Chong V. Screening for age-related macular degeneration using nonstereo digital fundus photographs. Eye 2006; 20: 471-475.

8 D'Souza Y, O'Toole L, Tufail A. The role of telemedicine in improving the referral service for consideration of PDT treatment in age related macular degeneration in a tertiary referral center. Poster exhibit no. 239. Royal College of Ophthalmologists Annual Congress 2006, Manchester, UK.

9 Hillman JG. Audit of elderly people's eye problems and non attendance at hospital eye service. BMJ 1994; 308: 953.

10 Scottish Health Service Costs (Costs Book) 2007. ISD Scotland.

11 Cannaby S, Westcott D, Pedersen CD, Voss H, Wanscher CE. The cost benefit of electronic patient referrals in Denmark: summary report. Stud Health Technol Inform 2004; 100: 238-245.

12 Bergmo TS. A cost-minimization analysis of a realtime teledermatology service in northern Norway. J Telemed Telecare 2000; 6: 273-277. 
13 Wootton R, Bloomer SE, Corbett R, Eedy DJ, Hicks N, Lotery HE et al Multicentre randomised control trial comparing real time teledermatology with conventional outpatient dermatological care: societal cost-benefit analysis. BMJ 2000; 320: 1252-1256.

14 White H, Gould D, Mills W, Brendish L. The Cornwall dermatology electronic referral and image-transfer project. J Telemed Telecare 1999; 5(Suppl 1): 85-86.

15 Northwood D. Referring to hospital consultants electronically: the Lewisham Hospital solution. Br J Healthcare Comput Info Manage 2001; 18(3): 30-32.
16 Kumar S, Yogesan K, Hudson B, Tay-Kearney M-L Constable IJ. Emergency eye care in rural Australia: role of internet. Eye 2006; 20: 1342-1344.

17 Kumar S, Tay-Kearney ML, Constable IJ, Yogesan K. Internet based ophthalmology service: impact assessment. Br J Ophthalmol 2005; 89(10): 1382-1383.

18 Chiang MF, Starren J, Du YE, Keenan JD, Schiff WM, Barile GR et al Remote image based retinopathy of prematurity diagnosis: a receiver operating characteristic analysis of accuracy. Br J Ophthalmol 2006; 90: 1292-1296.

19 Ingram DV, Culham LE. Ophthalmologists and optometrists -interesting times? Br J Ophthalmol 2001; 85(7): 769-770. 